\title{
A novel chaotic system with 11 piecewise linear functions and its analog circuit implementation
}

\author{
Jieyang Wang ${ }^{1}$, Jiawu Yu ${ }^{1}$, Huizhen Yan $^{1}$ \\ \{yujiawu_dlpu@sina.com\} \\ ${ }^{1}$ School of Information Science and Engineering, Dalian polytechnic University, Dalian, 116034, China
}

\begin{abstract}
In this paper, a new four-dimensional chaotic system with 11 piecewise linear functions is proposed in this paper. The dynamic characteristics of the four-dimensional chaotic system are analyzed through the Lyapunov exponential spectrum and bifurcation diagram. The state of the system under different bifurcation parameter changes is observed and recorded, and the exact parameters of the system entering the chaotic state are determined by the 10 test method. Numerical simulation results show that the system has entered chaos from the intermittent chaotic road, and the system has hidden attractors. Finally, the corresponding analog circuit is designed through system dynamics equations and circuit theory, which provides a complete theoretical basis for the system.
\end{abstract}

Keywords: 11 piecewise linear function; intermittent chaos road; hidden attractor; analog circuit implementation

\section{Introduction}

Chaos is a non-linear phenomenon widely existing in nature, and it is a basic form of motion [1-2]. The motion model of a dynamic system depends on two factors, one of which is the dynamic equation of the system, and the other is the initial conditions of the system [3-5]. For a deterministic system, when the system equation and initial conditions are determined, the result is determined, that is, the deterministic equation gets a definite solution, but in a chaotic system, even if the equation and initial conditions are determined, an uncertain solution will still be obtained [4-6]. In the chaotic system, three main roads are leading to chaos, namely the period-doubling bifurcation road, the PM-type intermittent road, and the pseudo-period road [78]. The way to enter chaos through PM intermittent roads is relatively rare compared to the other two, and it has a higher research value [9-11]. 
The PM type intermittent road was proposed by French scientists Y. Pomeau and P. Manneville, and it is also called the intermittent chaos road. The generation mechanism of PM-type intermittent roads is closely related to the tangential bifurcation [13-15]. This way occurs before the starting point of the tangential bifurcation, and the performance is that the periodic state and the chaotic state alternately appear with parameter changes [16-20]. When one of the parameters of the system is lower than a certain value, the system exhibits regular periodic motion [20-22]. When the parameters gradually change in a certain direction along the number axis, the system exhibits an obvious approximate periodic motion for a long time [23-24]. In the process of gradual change, the system has a brief chaotic behavior, and then it becomes a periodic motion [25]. This method is repeated continuously [26-28]. With the change of parameters, this sudden phenomenon becomes more and more frequent, and the approximate periodic motion becomes shorter and shorter [29-30]. Finally, the system enters a completely chaotic state [31].

Piecewise linear function is a kind of nonlinear function, which is usually used to make multiscroll chaotic systems and has important application value [32-34]. According to the different values of the slope of the function, piecewise linear functions can be divided into four categories [35-36]. By changing a parameter in the function, the number of wings of the multi-scroll chaotic system can be flexibly changed [37-38]. In this paper, by adding 11 piecewise linear functions to the chaotic system to increase the complexity, a new four-dimensional chaotic system with complex dynamics is obtained. The stability of the four-dimensional chaotic system is analyzed, and the results show that it is in a stable state, and the attractor of the system is hidden [39]. The complex dynamics and balance characteristics of the new system make it have a high application potential in military information transmission and confidential communication [40-42].

In this paper, the structure of the new 11 piecewise linear function and the attractor state of the system are introduced in the first part, and the equilibrium point and stability analysis of the new chaotic system are described in the second part. By using a bifurcation diagram, Lyapunov exponential spectrum, and a test method, the dynamic characteristics of this system are analyzed in the third part. The analog circuit corresponding to the new four-dimensional chaotic system is designed through Kirchhoff's law and circuit principle in the fourth part. The summary of the research results of this paper are presented in the fifth section.

\section{Novel four-dimensional chaotic system}

A new four-dimensional chaotic system with 11 piecewise linear functions can be expressed as 


$$
\left\{\begin{array}{l}
\dot{x}=-a(x+b y)+c x v \\
\dot{y}=-d(y-b x)+e y z \\
\dot{z}=-x^{2} z+x+H(x) \\
\dot{v}=-\left(1+h+100 y^{2}\right) v / 100+(h-1) / 10
\end{array}\right.
$$

among them, $\mathrm{H}(x)$ represents an 11 piecewise linear function, and piecewise function $\mathrm{H}(x)$ is an odd function, which is usually used to generate an even number of multi-scroll functions. In this paper, it is used to construct a new chaotic system. The construction method greatly increases the complexity of the chaotic system. The corresponding mathematical expression is shown in Formula 2, and the corresponding function image is shown in Figure 1.

$$
H(x)=m_{N} x+0.5 \sum_{i=1}^{N}\left(m_{i-1}-m_{i}\right)\left(\left|x+E_{i}\right|-\left|x-E_{i}\right|\right) .
$$

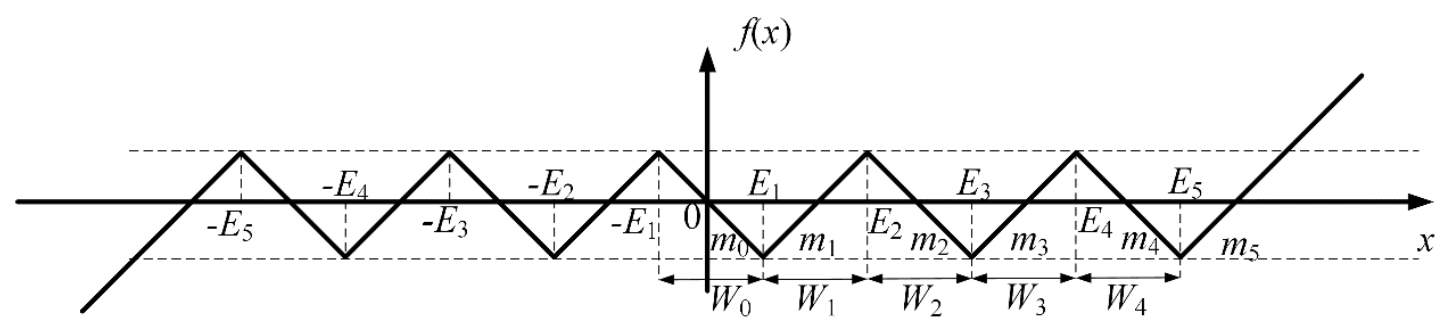

Fig 1 Function graph of 11 piecewise linear functions in the system

In Figure 1, the turning point value is $E_{i}$, the width of the linear interval is $W_{i}$, the slope of each interval is $m_{i}$, and the height of each turning point is equal. From this, the relationship between the width $W_{i}$ of each interval and the first turning point $E_{1}$ can be expressed as

$$
\begin{gathered}
W_{0}=2 \frac{\left|m_{0}\right|}{\left|m_{0}\right|} E_{1} \\
\vdots \\
W_{1}=2 \frac{\left|m_{0}\right|}{\left|m_{1}\right|} E_{1} \\
W_{i}=2 \frac{\left|m_{0}\right|}{\left|m_{i}\right|} E_{1}
\end{gathered}
$$

in formula $3, i=0,1,2$, the slope $m_{i}$ of each interval and the first turning point value $E_{1}$ are 
incorporated into the formula, and the calculation formula for turning point $E_{n}$ can be obtained as

$$
E_{n}=\left(1+2 \sum_{i=1}^{n-1} \frac{\left|m_{0}\right|}{\left|m_{i}\right|}\right) E_{1},
$$

expand it to get formula 5

$$
\left\{\begin{array}{l}
E_{2}=\left(1+2 \frac{\left|m_{0}\right|}{\left|m_{1}\right|}\right) E_{1} \\
E_{3}=\left(1+2 \frac{\left|m_{0}\right|}{\left|m_{1}\right|}+2 \frac{\left|m_{0}\right|}{\left|m_{2}\right|}\right) E_{1} \\
E_{4}=\left(1+2 \frac{\left|m_{0}\right|}{\left|m_{1}\right|}+2 \frac{\left|m_{0}\right|}{\left|m_{2}\right|}+2 \frac{\left|m_{0}\right|}{\left|m_{3}\right|}\right) E_{1} \\
E_{5}=\left(1+2 \frac{\left|m_{0}\right|}{\left|m_{1}\right|}+2 \frac{\left|m_{0}\right|}{\left|m_{2}\right|}+2 \frac{\left|m_{0}\right|}{\left|m_{3}\right|}+2 \frac{\left|m_{0}\right|}{\left|m_{4}\right|}\right) E_{1}
\end{array}\right.
$$

the specific expression of the 11 piecewise linear functions can be expressed as

$$
\begin{aligned}
H(x)=m_{5} x & +0.5\left(m_{0}-m_{1}\right)\left(\left|x+E_{1}\right|-\left|x-E_{1}\right|\right) \\
& +0.5\left(m_{1}-m_{2}\right)\left(\left|x+E_{2}\right|-\left|x-E_{2}\right|\right)+0.5\left(m_{2}-m_{3}\right)\left(\left|x+E_{3}\right|-\left|x-E_{3}\right|\right) \\
& +0.5\left(m_{3}-m_{4}\right)\left(\left|x+E_{4}\right|-\left|x-E_{4}\right|\right)+0.5\left(m_{4}-m_{5}\right)\left(\left|x+E_{5}\right|-\left|x-E_{5}\right|\right) \\
= & f_{0}(x)+f_{1}(x)+f_{2}(x)+f_{3}(x)+f_{4}(x)+f_{5}(x)
\end{aligned}
$$

When the system parameters take $a=4.5, b=1.6, c=19.5, d=9, e=6.7, h=8.1 . m_{0}=m_{2}=m_{4}=-0.5$, $m_{1}=m_{3}=m_{5}=0.3$, the first turning point value $E_{1}=0.4$. The initial value is $\left(\begin{array}{llll}0.1 & 0.1 & 0.10 .1\end{array}\right)$, when the step length is $0.01 \mathrm{~s}$, the Lyapunov exponent of the system is $(0.3588,0.0063,-1.1288$, 4.9655), and the system phase diagram is shown in Figure 2, the time domain diagram of the system is shown in Figure 3. 


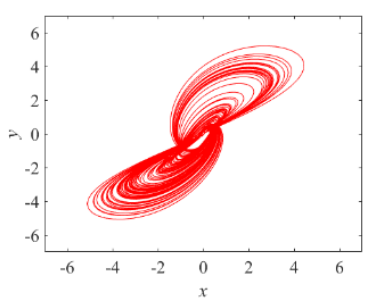

(a)

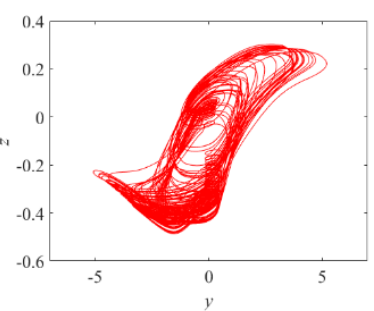

(d)

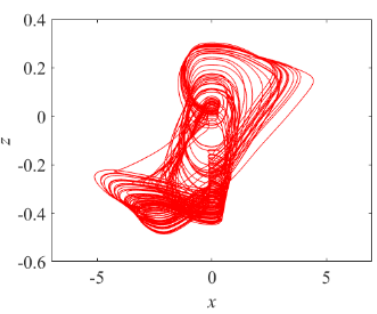

(b)

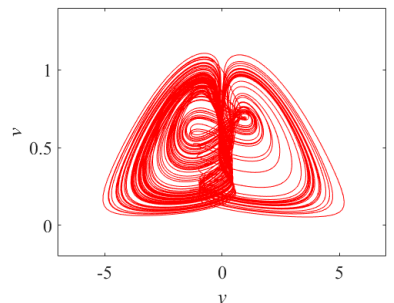

(e)

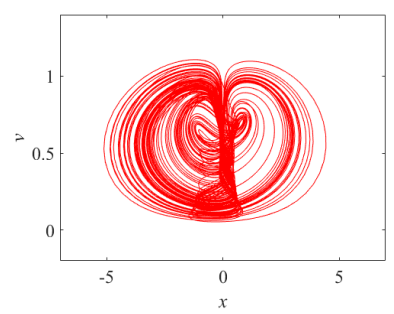

(c)

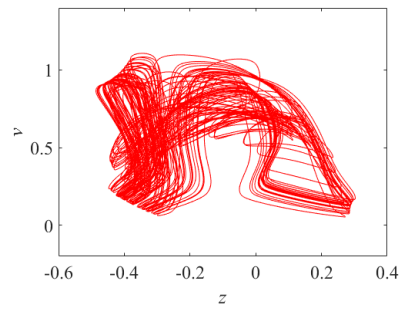

(f)

Fig 2 The phase diagram of the system, (a) $x-y$ plane, (b) $x-z$ plane, (c) $x$-v plane, (d) $y-z$ plane, (e) $y-v$ plane, (f) $z-v$ plane

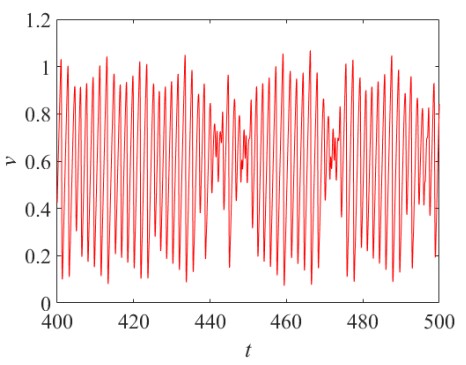

(a)

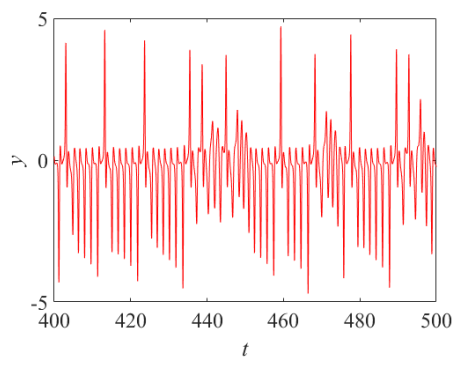

(c)

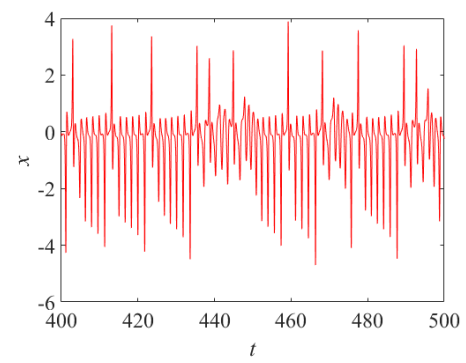

(b)

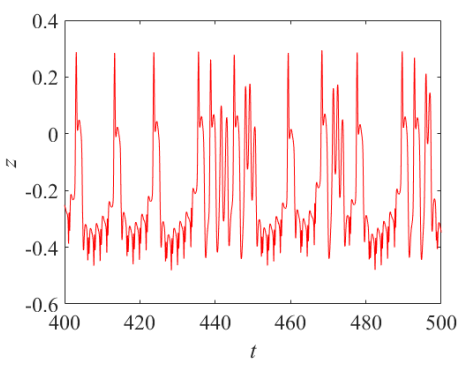

(d)

Fig 3 Time domain diagram of the system, (a) $t-x$ plane, (b) $t-y$ plane, (c) $t-z$ plane, (d) $t-v$ plane 


\section{Balance point analysis of system}

The divergence of the four-dimensional chaotic system is

$$
\begin{aligned}
\nabla V & =\frac{\partial \dot{x}}{\partial x}+\frac{\partial \dot{y}}{\partial y}+\frac{\partial \dot{z}}{\partial z}+\frac{\partial \dot{v}}{\partial v} \\
& =-a+c v-d+e z-x^{2}-\left(1+h+100 y^{2}\right) / 100
\end{aligned}
$$

when $-a+c v-d+e z-x^{2}-\left(1+h+100 y^{2}\right) / 100<0$, the system is dissipative, and the volume of system is 0 , when $t$ approaches infinity. Let the parameters $a=4.5, b=1.6, c=19.5, d=9, e=6.7, h=8.1$. $m_{0}=m_{2}=m_{4}=-0.5, m_{1}=m_{3}=m_{5}=0.3, E_{1}=0.4$. When the initial value is $\left(\begin{array}{llll}0.1 & 0.1 & 0.1 & 0.1\end{array}\right)$, the divergence is less than 0 , the system at this time is dissipative, and has the conditions to produce chaos.

Let the differential equations of the system be equal to zero, that is, when the system equation takes $\dot{x}=\dot{y}=\dot{z}=\dot{v}=0$, the equilibrium point of the system can be obtained.

$$
\left\{\begin{array}{l}
-a(x+b y)+c x v=0 \\
-d(y-b x)+e y z=0 \\
-x^{2} z / 100+x+H(x)=0 \\
-\left(1+h+100 y^{2}\right) v / 100+(h-1) / 10=0
\end{array},\right.
$$

it can be easily found that the equilibrium point of the system is $P(0,0,1.3303,7.8022)$. Linearize the system at point $P$, and the Jacobian matrix of the system is

$$
\left[\begin{array}{cccc}
c v-a & -a b & 0 & c x \\
b d & e z-d & e y & 0 \\
P(x) & 0 & -x^{2} & 0 \\
0 & -2 v y & 0 & -y^{2}-h / 100-0.01
\end{array}\right]
$$

among them, expand $\mathrm{P}(x)$ to get formula 10

$$
\begin{aligned}
P(x)= & +\left(\operatorname{sgn}\left(E_{1}+x\right)+0.5 \operatorname{sgn}\left(E_{1}-x\right)\right)\left(m_{0}-m_{1}\right)-2 x z \\
& +\left(\operatorname{sgn}\left(E_{2}+x\right)+0.5 \operatorname{sgn}\left(E_{2}-x\right)\right)\left(m_{1}-m_{2}\right)+m_{5} \\
& +\left(\operatorname{sgn}\left(E_{3}+x\right)+0.5 \operatorname{sgn}\left(E_{3}-x\right)\right)\left(m_{2}-m_{3}\right)+1 \\
& +\left(\operatorname{sgn}\left(E_{4}+x\right)+0.5 \operatorname{sgn}\left(E_{4}-x\right)\right)\left(m_{3}-m_{4}\right) \\
& +\left(\operatorname{sgn}\left(E_{5}+x\right)+0.5 \operatorname{sgn}\left(E_{5}-x\right)\right)\left(m_{4}-m_{5}\right)
\end{aligned}
$$

By analyzing the Jacobian matrix, the characteristic polynomial of the system can be 
expressed as

$$
f(\lambda)=A_{0} \lambda^{4}+A_{1} \lambda^{3}+A_{2} \lambda^{2}+A_{3} \lambda^{1}+A_{4}
$$

according to the Rouss-Herwitz stability criterion, the system is stable under the following conditions.

$$
\begin{aligned}
& A_{n}>0 \\
& A_{1} A_{2}>A_{0} A_{3} \\
& A_{1} A_{2} A_{3}>A_{0} A_{3}^{2}+A_{1}^{2} A_{4}
\end{aligned}
$$

putting $A_{n}$ into formula 12 to calculate in turn shows that the system is stable according to the stability judgment. Therefore, all attractors in this system are hidden attractors.

\section{Dynamics analysis of system}

The bifurcation graph and Lyapunov exponent spectrum are important indicators to determine the state of the system. To obtain more accurate parameter values when the system enters chaos, the 10 test method is used for system analysis. This test method can accurately determine the parameter values when the system enters chaos.

Let the system parameters $a=4.5, b=1.6, d=9, e=6.7, h=8.1 . m_{0}=m_{2}=m_{4}=-0.5, m_{1}=m_{3}=m_{5}=0.3$,

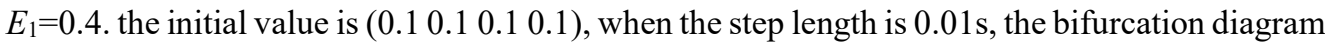
and Lyapunov index spectrum obtained according to the change of $c \in(18,21)$ are shown in Figure 4. According to Figure 4, as the parameter $c$ decreases from 21, the system begins to produce a transient state of chaos. When the parameter value is $c=20.909$, the system enters chaos for the first time, the Lyapunov exponent is $(0.0996,-0.0165,-1.3122,-6.2662)$, and the duration gradually becomes longer, and finally, it enters a continuous chaotic state. When $c=18.468$, the system begins to show obvious period-doubling bifurcation. At this time, the Lyapunov exponent is $(0.0133,-0.0801,-0.7587,-3.8666)$, and the system gradually enters a new chaotic state. The 10 test chart when the system enters chaos and periodic state for the first time is shown in Fig. 5. 


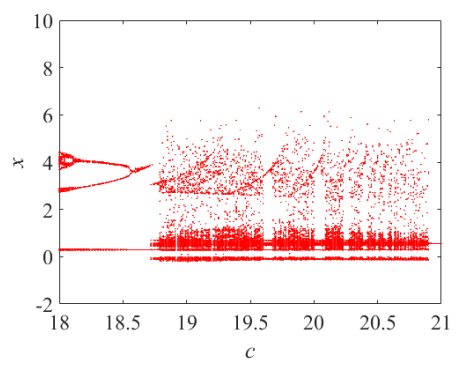

(a)

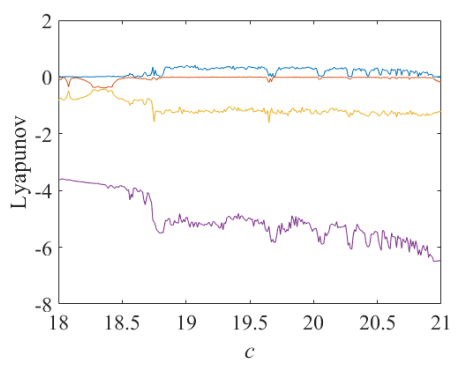

(b)

Fig 4 The Lyapunov index spectrum and bifurcation diagram of the system when $c$ changes, (a) the bifurcation diagram of the system, (b) the Lyapunov index diagram of the system

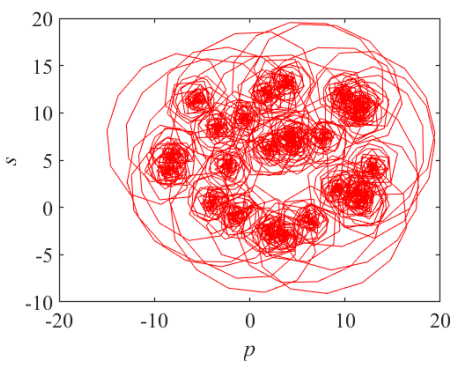

(a)

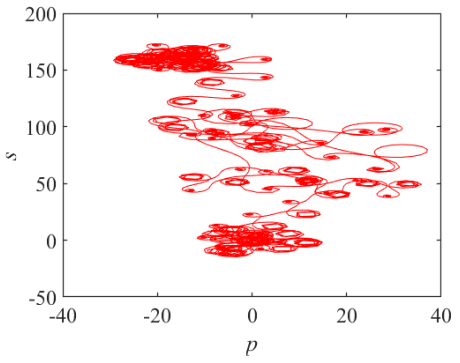

(b)

Fig 5 The 10 test chart of the system when $c$ changes, (a) $c=20.90$, (b) $c=18.468$

Let the system parameters $a=4.5, b=1.6, c=19.5, e=6.7, h=8.1 . m_{0}=m_{2}=m_{4}=-0.5, m_{1}=m_{3}=m_{5}=0.3$,

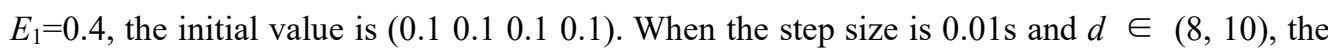
bifurcation graph and Lyapunov index spectrum of the system are shown in Figure 6. And, Figure 7 is the 10 test chart when the system enters the chaotic state and the periodic state for the first time under different values of the parameter $d$. With the increase of $\mathrm{d}$, when $d=8.224$, the system enters a chaotic state, at this time, the Lyapunov index of the system is $(0.2243$, $0.0034,-1.2404,-5.3852)$. When $d \in(8.861,8.889)$, the system behaves as a periodic state. When $d=8.623$, the system is in the periodic window, and the Lyapunov index of the system at this time is $(0.02565,-0.03074,-1.371,-5.538)$. When $d>9.719$, the system enters a periodic state. At this time, the Lyapunov exponent is $(0.0171,-0.0763,-0.6976,-4.4898)$. 


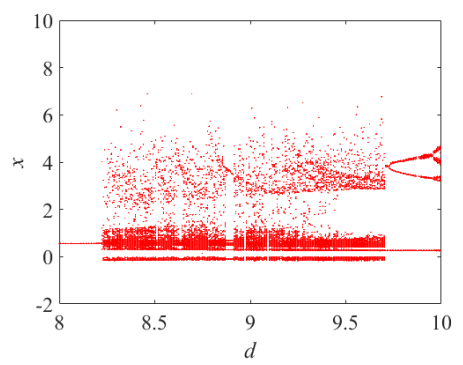

(a)

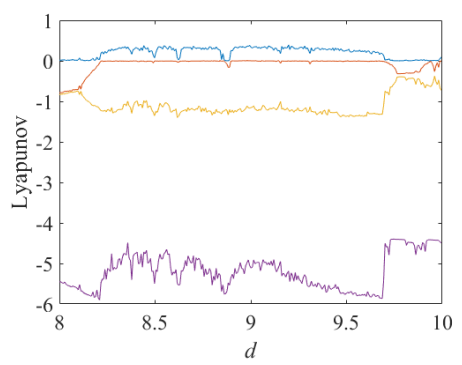

(b)

Fig 6 The Lyapunov index spectrum and bifurcation diagram of the system when $d$ changes, (a) the bifurcation diagram of the system, (b) the Lyapunov index diagram of the system

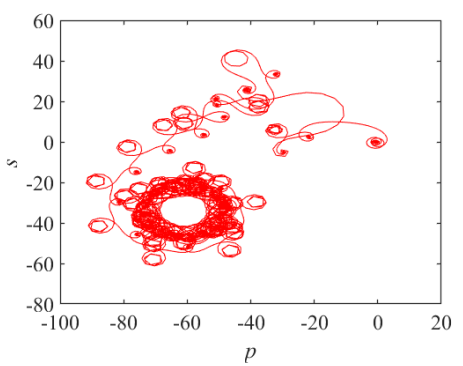

(a)

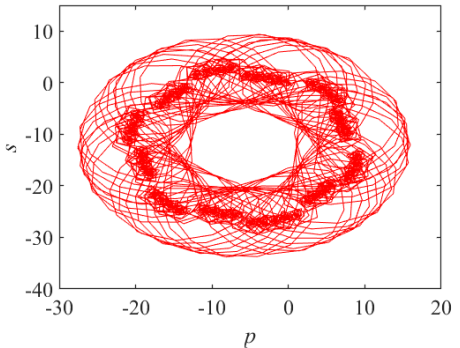

(b)

Fig 7 The 10 test chart of the system when $d$ changes, (a) $d=20.90$, (b) $d=18.468$

\section{Analog circuit design of system}

In order to perfect the theory of the system, an analog circuit corresponding to the new system shown in formula 13 is designed. The analog circuit is composed of the main arithmetic circuit and an 11 piecewise linear function circuit. The complete circuit consists of resistors, capacitors, multipliers, and operational amplifiers.

$$
\left\{\begin{array}{l}
\dot{x}=-a(x+b y)+c x v \\
\dot{y}=-d(y-b x)+e y z \\
\dot{z}=-x^{2} z+x+H(x) \\
\dot{v}=-\left(1+h+100 y^{2}\right) v / 100+(h-1) / 10
\end{array} .\right.
$$

Dimension formula 13 to obtain the equation system shown in formula 14. The first and second differential equations in the equation system are coupled with each other, corresponding to the two sets of circuits in Figure 8 (a) and (b), the piecewise linear function is added in the third 
differential equation.

$$
\left\{\begin{array}{l}
C_{1} V_{x}=\frac{V_{x}}{R_{1}}+\frac{V_{y}}{R_{2}}+\frac{R_{4} g_{1} V_{x} V_{v}}{R_{3} R_{5}} \\
C_{2} V_{y}=\frac{V_{y}}{R_{6}}+\frac{R_{7} V_{x}}{R_{8} R_{9}}+\frac{R_{10} g_{2} V_{y} V_{z}}{R_{11} R_{12}} \\
C_{3} V_{z}=\frac{g_{3} g_{4} V_{x}^{2} V_{z}}{R_{13}}+\frac{R_{14} V_{x}}{R_{15} R_{16}}+\frac{R_{17} H\left(V_{x}\right)}{R_{18} R_{19}} \\
C_{4} V_{v}=\frac{V_{v}}{R_{20}}+\frac{V_{v}}{R_{21}}+\frac{g_{5} g_{6} V_{v} V_{y}^{2}}{R_{22}}+\frac{V}{R_{23}}
\end{array},\right.
$$

among them, $v_{x}, v_{y}, v_{z}, v_{v}$ are the four circuit variables of the simulation circuit, $g_{1}, g_{2}, g_{3}, g_{4}, g_{5}$, $g_{6}$ are the amplification coefficients of the multiplier, and $V$ represents the constant term. According to the logical relationship shown in formula 14, combined with Kirchhoff's law and circuit principle, the analog circuit corresponding to system 13 can be obtained.
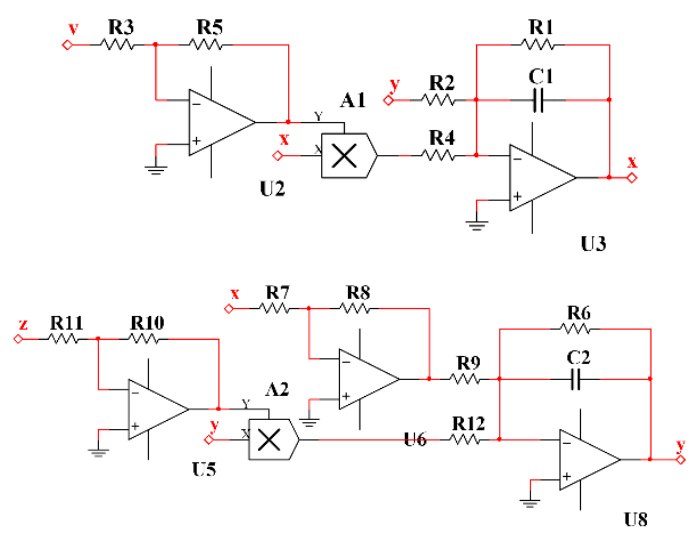

(a)

(b)

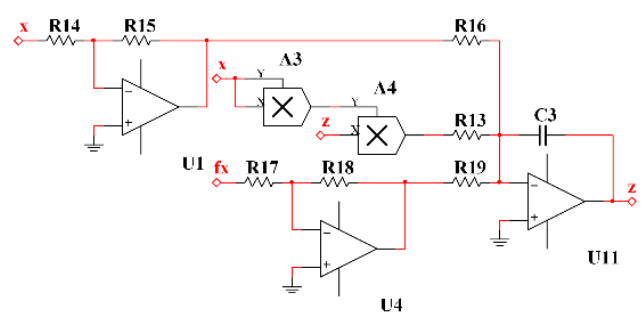

(c)

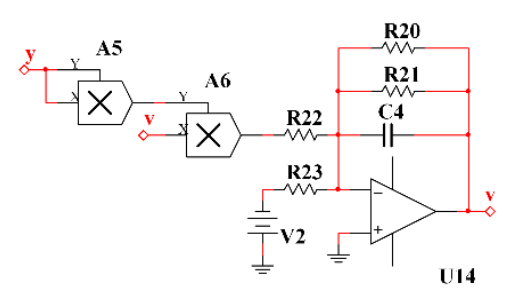

(d) 


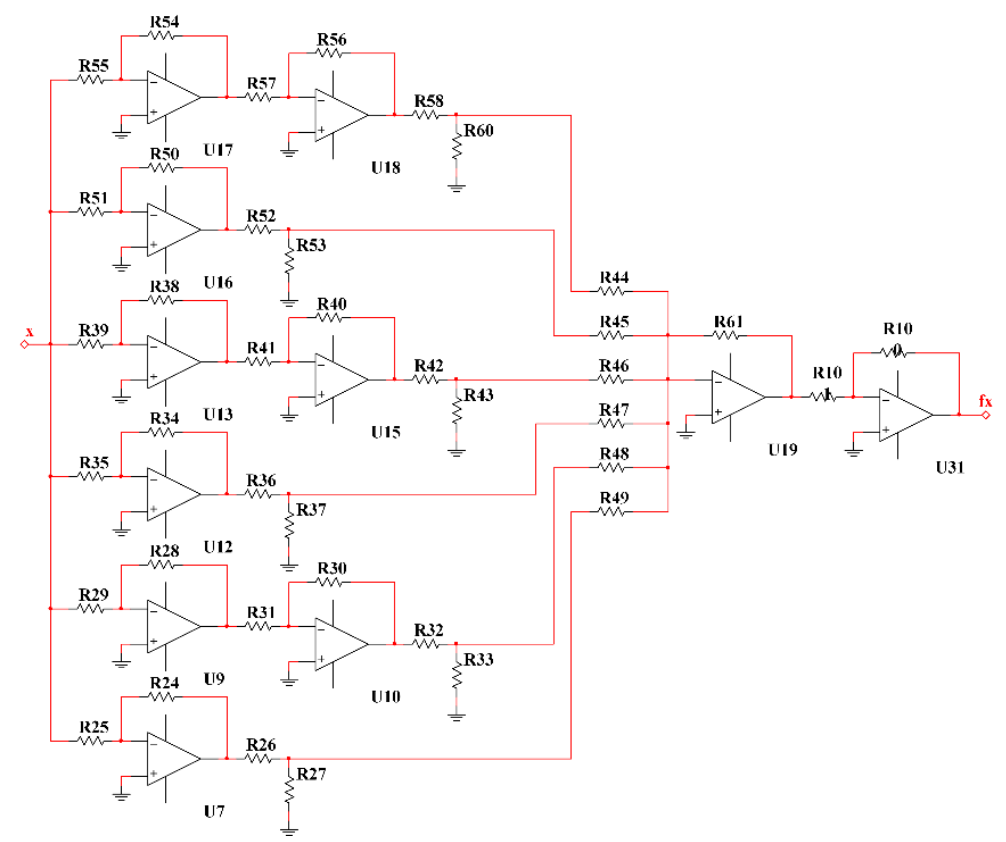

(e)

Fig 8 The simulation circuit corresponding to the four-dimensional chaotic system, (a) the first differential equation (b) the second differential equation (c) the third differential equation (d) the fourth differential equation (e) 11 piecewise linear function

\section{Conclusion}

In this paper, by introducing 11 piecewise linear functions to the chaotic system, a new fourdimensional chaotic system with complex dynamic behavior is proposed. Methods such as bifurcation diagram, Lyapunov exponent spectrum, phase diagram, and 10 test are used to analyze the system. Numerical simulation results show that due to the introduction of 11 piecewise linear functions, the system has hidden attractors and complex dynamics. For example, when some parameters are fixed, when the parameter $\mathrm{c}$ becomes smaller, the system shows obvious intermittent chaos. The chaotic state of the system appears more and more frequently and lasts longer and longer until it finally enters the chaotic zone completely. Finally, the analog circuit diagram corresponding to the system is designed using the circuit principle, which provides a complete theoretical basis for scholars to study the system. The complex dynamics of the system provide a good prospect for its practical application.

\section{Acknowledgments}

This work was supported by Provincial Natural Science Foundation of Liaoning (Grant Nos. 
2020-MS-274); National Natural Science Foundation of China (Grant Nos. 62061014).

\section{Author contributions}

Jieyang Wang designed and carried out experiments, data analyzed and manuscript wrote. Jiawu Yu made the theoretical guidance for this paper. Huizhen Yan improved the algorithm. All authors reviewed the manuscript.

\section{Conflicts of Interest}

No conflicts of interests about the publication by all authors.

\section{References}

[1] Tigan G , Opri D . Analysis of a 3D chaotic system[J]. Chaos Solitons \& Fractals, 2008, 36(5):1315-1319.

[2] Qi G, Du S, Chen G, et al. On a four-dimensional chaotic system[J]. Chaos Solitons \& Fractals, 2005, 23(5):1671-1682.

[3] Zhang F D . The Synchronization of a Fractional-Order Chaotic System[J]. Advanced Materials Research, 2013, 655-657:1488-1491.

[4] $\quad \mathrm{Lu} \mathrm{J}, \mathrm{Wu} X$, Jinhu Lü. Synchronization of a unified chaotic system and the application in secure communication[J]. Physics Letters A, 2002, 305(6):365-370.

[5] Chen, Shi-Hua, Zhao, et al. Parameter identification and synchronization of an uncertain Chen chaotic system via adaptive control[J]. Chinese Physics, 2002, 11(6):543-543.

[6] Li Y, Yang B J , Badal J , et al. Chaotic system detection of weak seismic signals[J]. Geophysical Journal International(3):1493-1522.

[7] Sun, H, Cao. Chaos control and synchronization of a modified chaotic system[J]. CHAOS SOLITONS AND FRACTALS, 2008.

[8] Muthukumar P , Balasubramaniam P , Ratnavelu K . Synchronization and an application of a novel fractional order King Cobra chaotic system[J]. Chaos, 2014, 24(3):10000-968.

[9] Kodama H , Sato S , Honda K . Reconsideration of the renormalization-group theory on intermittent chaos[J]. Physics Letters A, 1991, 157(6-7):354-356.

[10] Ditto W L , Rauseo S N , Spano M L . Experimental control of chaos[J]. Controlling Chaos, 1996, 65(26):105-107.

[11] Cosenza M G , Alvarez-Llamoza O , Ponce G A . Lyapunov exponent for type-III intermittent chaos[J]. Communications in Nonlinear Science \& Numerical Simulation, 2010, 15(9):24312435 .

[12] Abarbanel H D I, Rulkov N F, Sushchik M M . Generalized synchronization of chaos: The auxiliary system approach[J]. Physical Review E Statistical Physics Plasmas Fluids \& Related 
Interdisciplinary Topics, 1996, 53(5):4528-4535.

[13] Tse C K , Zhou Y, Lau F C M , et al. Intermittent chaos and subharmonics in switching power supplies[C]// Circuits and Systems, 2003. ISCAS '03. Proceedings of the 2003 International Symposium on. IEEE, 2003.

[14] Gabriel Pérez, Cerdeira H A. Extracting Messages Masked by Chaos[J]. Physical Review Letters, 1995, 74(11):1970-1973.

[15] Hisao O , Hazime M , Shoichi K . Global Spectral Structures of Type III Intermittent Chaos[J]. Progress of Theoretical Physics(3):784-801.

[16] N, Mori, T, et al. Scaling Structures and Statistical Mechanics of Type I Intermittent Chaos[J]. Progress of Theoretical Physics, 1989, 81(1).

[17] Njougouo T, Simo G R, Louodop P, et al. Effects of intermittent coupling on synchronization[J]. Chaos Solitons \& Fractals, 2020, 139:110082.

[18] Koronovskii A A , Moskalenko O I , Pivovarov A A, et al. Intermittent route to generalized synchronization in bidirectionally coupled chaotic oscillators[J]. Chaos, 2020, 30(8):083133.

[19] Pierre Bergé, Pomeau Y, Vidal C . Order within chaos: towards a deterministic approach to turbulence. Transl. from the French[J]. 1987.

[20] Jahanshahi H, Onofre Orozco-López, Munoz-Pacheco J M , et al. Simulation and experimental validation of a non-equilibrium chaotic system[J]. Chaos Solitons \& Fractals, 2021, 143:110539.

[21] Yang Y, Wang L, Duan S, et al. Dynamical analysis and image encryption application of a novel memristive hyperchaotic system[J]. Optics \& Laser Technology, 2021, 133:106553.

[22] Khan A , Jahanzaib L S , Trikha P . Changing Dynamics of the First, Second and Third Approximations of the Exponential Chaotic System and Their Application in Secure Communication Using Synchronization[J]. International Journal of Applied and Computational Mathematics, 2021, 7(1).

[23] Qi G , Gou T, Hu J , et al. Breaking of integrability and conservation leading to Hamiltonian chaotic system and its energy-based coexistence analysis[J]. Chaos, 2021, 31(1):013101.

[24] Kubba Z M J , Hoomod H K . Modified PRESENT Encryption algorithm based on new 5D Chaotic system[J]. IOP Conference Series: Materials Science and Engineering, 2020, 928(3):032023 (8pp).

[25] Li H , Deng L , Gu Z . A Robust Image Encryption Algorithm Based on a 32-bit Chaotic System[J]. IEEE Access, 2020, 8:30127-30151.

[26] Niu Y, Sun X, Zhang C , et al. Anticontrol of a Fractional-Order Chaotic System and Its Application in Color Image Encryption[J]. Mathematical Problems in Engineering, 2020, 2020.

[27] J. C , Antoranz, and, et al. Intermittent incommensurate chaos[J]. Physica D: Nonlinear Phenomena, 1985, 16(2):184-202. 
[28] Goldhirsch I , Noskowicz S H , Schuss Z . Band-edge localization as intermittent chaos[J]. Physical Review B Condensed Matter, 1993, 47(4):1918.

[29] Ohtaki Y, Takara K, Onodera F , et al. A SIMPLE MODEL OF INTERMITTENT CHAOS[J]. 2015.

[30] Takeshi K , Yoshiro K . Intermittent Chaos Generated by Logarithmic Map[J]. Progress of Theoretical Physics(3):581-586.

[31] MORI, Hazime, OKAMOTO, et al. Global Spectral Structures of Intermittent Chaos[J]. Ippj Rev, 1986.

[32] Shaobo H, Kehui S, Huihai W. Modified multiscale permutation entropy algorithm and its application for multiscroll chaotic systems[J]. Complexity, 2016:52-58.

[33] Liu S, Wei Y, Liu J , et al. Multi-Scroll Chaotic System Model and Its Cryptographic Application[J]. International Journal of Bifurcation and Chaos, 2020.

[34] Shao X, Chen F . The Projective Synchronization of Fractional-order Multi-scroll Chaotic Systems[C]// 2015 4th International Conference on Sensors, Measurement and Intelligent Materials. 2016.

[35] Gui Z, Wu X, Lin Q . Finite Time Synchronization of Nonautonomous Multiscroll Chaotic Systems $[\mathrm{C}] / /$ International Workshop on Chaos-fractals Theories \& Applications. 0. Senouci, Abdelkader, Boukabou, et al. Fuzzy modeling, stabilization and synchronization of multi-scroll chaotic systems.[J]. Optik - International Journal for Light \& Electron Optics, 2016.

[36] A A K M , B S D , A V K Y . Finite-time synchronization of multi-scroll chaotic systems with sigmoid non-linearity and uncertain terms - ScienceDirect[J]. Chinese Journal of Physics, 2020.

[37] Khelifa M A, Boukabou A. Design of an intelligent prediction-based neural network controller for multi-scroll chaotic systems[J]. Applied Intelligence, 2016, 45(3):1-15.

[38] Yuan F, Wang G, Wang X . Extreme multistability in a memristor-based multi-scroll hyperchaotic system[J]. Chaos, 2016, 26(7):507-519. Xiulan, Zhang, Xin, et al. Generalized synchronization for multidirectional saturated multi-scroll chaotic systems[J]. International Journal of Applied Mathematics \& Statistics, 2013.

[39] Khelifa, Mohammed, Amin, et al. Design of an intelligent prediction-based neural network controller for multi-scroll chaotic systems[J]. Applied Intelligence: The International Journal of Artificial Intelligence, Neural Networks, and Complex Problem-Solving Technologies, 2016.

[40] Cong W , Hong-Li Z, University X . Parameter identification for fractional-order multi-scroll chaotic systems based on original dual-state transition algorithm[J]. Acta Physica Sinica, 2016, 65(6). 


\section{Appendix}

The coefficient $A_{n}$ of the characteristic polynomial involved in the third part of this paper is shown in here.

$$
\begin{aligned}
& A_{0}=1 \\
& A_{1}=x^{2}+y^{2}+a+d+h / 100-c w-e z+1 / 100 \\
& A_{2}=a / 100+d / 100+x^{2} y^{2}+a d+a h / 100+d h / 100-c w / 100 \\
& -e z / 100+a x^{2}+a y^{2}+d x^{2}+d y^{2}+h x^{2} / 100+x^{2} / 100-c w x^{2} \\
& -c w y^{2}-e x^{2} z-e y^{2} z-c d w-a e z-c h w / 100-e h z / 100+a b^{2} d+c e w z \\
& A_{3}=(a d) / 100+\left(a x^{2}\right) / 100+\left(d x^{2}\right) / 100+\left(d h x^{2}\right) / 100 \\
& -\left(c w x^{2}\right) / 100-\left(e x^{2} z\right) / 100+a x^{2} y^{2}+d x^{2} y^{2}+(a d h) / 100 \\
& -(c d w) / 100-(a e z) / 100+\left(a b^{2} d\right) / 100+a d x^{2}+a d y^{2} \\
& +\left(a h x^{2}\right) / 100+a b e y-(c d h w) / 100-(a e h z) / 100+(c e w z) / 100 \\
& +\left(a b^{2} d h\right) / 100-c d w x^{2}-c d w y^{2}-a e x^{2} z-a e y^{2} z-\left(c h w x^{2}\right) / 100 \\
& -\left(e h x^{2} z\right) / 100+a b^{2} d x^{2}+a b^{2} d y^{2}-c w x^{2} y^{2}-e x^{2} y^{2} z+a b e m 5 y \\
& +(\operatorname{cehwz}) / 100+\operatorname{cewx} x^{2} z+\operatorname{cewy}^{2} z+(\operatorname{abem} 0 y \operatorname{sign}(E 1-x)) / 2 \\
& -(\operatorname{abem} 1 y \operatorname{sign}(E 1-x)) / 2+(\operatorname{abem} 1 y \operatorname{sign}(E 2-x)) / 2 \\
& -(\operatorname{abem} 2 y \operatorname{sign}(E 2-x)) / 2+(\operatorname{abem} 2 y \operatorname{sign}(E 3-x)) / 2 \\
& -(\operatorname{abem} 3 y \operatorname{sign}(E 3-x)) / 2+(\operatorname{abem} 3 y \operatorname{sign}(E 4-x)) / 2 \\
& -(\operatorname{abem} 4 y \operatorname{sign}(E 4-x)) / 2+(\operatorname{abem} 4 y \operatorname{sign}(E 5-x)) / 2 \\
& -(\operatorname{abem} 5 y \operatorname{sign}(E 5-x)) / 2+2 b c d w x y-2 a b e x y z \\
& +(\operatorname{abem} 0 y \operatorname{sign}(E 1+x)) / 2-(\operatorname{abem} 1 y \operatorname{sign}(E 1+x)) / 2 \\
& +(\operatorname{abem} 1 y \operatorname{sign}(E 2+x)) / 2-(\operatorname{abem} 2 y \operatorname{sign}(E 2+x)) / 2 \\
& +(\operatorname{abem} 2 y \operatorname{sign}(E 3+x)) / 2-(\operatorname{abem} 3 y \operatorname{sign}(E 3+x)) / 2 \\
& +(\operatorname{abem} 3 y \operatorname{sign}(E 4+x)) / 2-(\operatorname{abem} 4 y \operatorname{sign}(E 4+x)) / 2 \\
& +(\operatorname{abem} 4 y \operatorname{sign}(E 5+x)) / 2-(\operatorname{abem} 5 y \operatorname{sign}(E 5+x)) / 2
\end{aligned}
$$




$$
\begin{aligned}
& A_{4}=\left(a d x^{2}\right) / 100+(\text { abey }) / 100+a b e y^{3}+\left(a d h x^{2}\right) / 100-\left(c d w x^{2}\right) / 100 \\
& -\left(a e x^{2} z\right) / 100+\left(a b^{2} d x^{2}\right) / 100+a d x^{2} y^{2}+\left(a b^{2} d h x^{2}\right) / 100-c d w x^{2} y^{2} \\
& -a e x^{2} y^{2} z+(\text { abehy }) / 100+\left(\text { abem5y) } / 100+a b^{2} d x^{2} y^{2}+a b e m 5 y^{3}\right. \\
& -\left(c d h w x^{2}\right) / 100-\left(a e h x^{2} z\right) / 100+2 c e w x y^{2}+\left(c e w x^{2} z\right) / 100+2 b c d w x^{3} y \\
& -2 a b e x y^{3} z+\left(\operatorname{ceh} w x^{2} z\right) / 100+2 \operatorname{cem} 5 w x y^{2}+(\operatorname{abem} 0 y \operatorname{sign}(E 1-x)) / 200 \\
& -(\operatorname{abem} 1 y \operatorname{sign}(E 1-x)) / 200+(\operatorname{abem} 1 y \operatorname{sign}(E 2-x)) / 200 \\
& -(\operatorname{abem} 2 y \operatorname{sign}(E 2-x)) / 200+(\operatorname{abem} 2 y \operatorname{sign}(E 3-x)) / 200 \\
& -(\operatorname{abem} 3 y \operatorname{sign}(E 3-x)) / 200+(\operatorname{abem} 3 y \operatorname{sign}(E 4-x)) / 200 \\
& -(\operatorname{abem} 4 y \operatorname{sign}(E 4-x)) / 200+(\operatorname{abem} 4 y \operatorname{sign}(E 5-x)) / 200 \\
& -(\operatorname{abem} 5 y \operatorname{sign}(E 5-x)) / 200+\left(\operatorname{abem} 0 y^{3} \operatorname{sign}(E 1+x)\right) / 2 \\
& -\left(\operatorname{abem} 1 y^{3} \operatorname{sign}(E 1+x)\right) / 2+\left(\operatorname{abem} 1 y^{3} \operatorname{sign}(E 2+x)\right) / 2 \\
& -\left(\operatorname{abem} 2 y^{3} \operatorname{sign}(E 2+x)\right) / 2+\left(\operatorname{abem} 2 y^{3} \operatorname{sign}(E 3+x)\right) / 2 \\
& -\left(\operatorname{abem} 3 y^{3} \operatorname{sign}(E 3+x)\right) / 2+\left(\operatorname{abem} 3 y^{3} \operatorname{sign}(E 4+x)\right) / 2 \\
& -\left(\operatorname{abem} 4 y^{3} \operatorname{sign}(E 4+x)\right) / 2+\left(\operatorname{abem} 4 y^{3} \operatorname{sign}(E 5+x)\right) / 2 \\
& -\left(\operatorname{abem} 5 y^{3} \operatorname{sign}(E 5+x)\right) / 2+\left(\operatorname{abem} 0 y^{3} \operatorname{sign}(E 1-x)\right) / 2 \\
& -\left(\operatorname{abem} 1 y^{3} \operatorname{sign}(E 1-x)\right) / 2+\left(\operatorname{abem} 1 y^{3} \operatorname{sign}(E 2-x)\right) / 2 \\
& -\left(\operatorname{abem} 2 y^{3} \operatorname{sign}(E 2-x)\right) / 2+\left(\operatorname{abem} 2 y^{3} \operatorname{sign}(E 3-x)\right) / 2 \\
& -\left(\operatorname{abem} 3 y^{3} \operatorname{sign}(E 3-x)\right) / 2+\left(\operatorname{abem} 3 y^{3} \operatorname{sign}(E 4-x)\right) / 2 \\
& -\left(\operatorname{abem} 4 y^{3} \operatorname{sign}(E 4-x)\right) / 2+\left(\operatorname{abem} 4 y^{3} \operatorname{sign}(E 5-x)\right) / 2 \\
& -\left(\operatorname{abem} 5 y^{3} \operatorname{sign}(E 5-x)\right) / 2-3 c e w x^{2} y^{2} z+(\text { abehm } 5 y) / 100 \\
& -(\text { abexyz }) / 50+(\operatorname{abem} 0 y \operatorname{sign}(E 1+x)) / 200 \\
& -(\operatorname{abem} 1 y \operatorname{sign}(E 1+x)) / 200+(\operatorname{abem} 1 y \operatorname{sign}(E 2+x)) / 200 \\
& -(\operatorname{abem} 2 y \operatorname{sign}(E 2+x)) / 200+(\operatorname{abem} 2 y \operatorname{sign}(E 3+x)) / 200 \\
& -(\operatorname{abem} 3 y \operatorname{sign}(E 3+x)) / 200+(\operatorname{abem} 3 y \operatorname{sign}(E 4+x)) / 200 \\
& -(\operatorname{abem} 4 y \operatorname{sign}(E 4+x)) / 200+(\operatorname{abem} 4 y \operatorname{sign}(E 5+x)) / 200
\end{aligned}
$$




$$
\begin{aligned}
& -(\operatorname{abem} 5 y \operatorname{sign}(E 5+x)) / 200+\operatorname{cem} 0 w x y^{2} \operatorname{sign}(E 1-x) \\
& -\operatorname{cem} 1 w x y^{2} \operatorname{sign}(E 1-x)+\operatorname{cem} 1 w x y^{2} \operatorname{sign}(E 2-x) \\
& -\operatorname{cem} 2 w x y^{2} \operatorname{sign}(E 2-x)+\operatorname{cem} 2 w x y^{2} \operatorname{sign}(E 3-x) \\
& -\operatorname{cem} 3 w x y^{2} \operatorname{sign}(E 3-x)+\operatorname{cem} 3 w x y^{2} \operatorname{sign}(E 4-x) \\
& -\operatorname{cem} 4 w x y^{2} \operatorname{sign}(E 4-x)+\operatorname{cem} 4 w x y^{2} \operatorname{sign}(E 5-x) \\
& -\operatorname{cem} 5 w x y^{2} \operatorname{sign}(E 5-x)-(\text { abehxyz }) / 50 \\
& +(\operatorname{abehm} 0 y \operatorname{sign}(E 1+x)) / 200-(\operatorname{abehm} 1 y \operatorname{sign}(E 1+x)) / 200 \\
& +(\operatorname{abehm} 1 y \operatorname{sign}(E 2+x)) / 200-(\operatorname{abehm} 2 y \operatorname{sign}(E 2+x)) / 200 \\
& +(\operatorname{abehm} 2 y \operatorname{sign}(E 3+x)) / 200-(\operatorname{abehm} 3 y \operatorname{sign}(E 3+x)) / 200 \\
& +(\operatorname{abehm} 3 y \operatorname{sign}(E 4+x)) / 200-(\operatorname{abehm} 4 y \operatorname{sign}(E 4+x)) / 200 \\
& +(\operatorname{abehm} 4 y \operatorname{sign}(E 5+x)) / 200-(\operatorname{abehm} 5 y \operatorname{sign}(E 5+x)) / 200 \\
& +(\operatorname{abehm} 0 y \operatorname{sign}(E 1-x)) / 200-(\operatorname{abehm} 1 y \operatorname{sign}(E 1-x)) / 200 \\
& +(\operatorname{abehm} 1 y \operatorname{sign}(E 2-x)) / 200-(\operatorname{abehm} 2 y \operatorname{sign}(E 2-x)) / 200 \\
& +(\operatorname{abehm} 2 y \operatorname{sign}(E 3-x)) / 200-(\operatorname{abehm} 3 y \operatorname{sign}(E 3-x)) / 200 \\
& +(\operatorname{abehm} 3 y \operatorname{sign}(E 4-x)) / 200-(\operatorname{abehm} 4 y \operatorname{sign}(E 4-x)) / 200 \\
& +(\operatorname{abehm} 4 y \operatorname{sign}(E 5-x)) / 200-(\operatorname{abehm} 5 y \operatorname{sign}(E 5-x)) / 200 \\
& +\operatorname{cem} 0 w x y^{2} \operatorname{sign}(E 1+x)-\operatorname{cem} 1 w x y^{2} \operatorname{sign}(E 1+x) \\
& +\operatorname{cem} 1 w x y^{2} \operatorname{sign}(E 2+x)-\operatorname{cem} 2 w x y^{2} \operatorname{sign}(E 2+x) \\
& +\operatorname{cem} 2 w x y^{2} \operatorname{sign}(E 3+x)-\operatorname{cem} 3 w x y^{2} \operatorname{sign}(E 3+x) \\
& +\operatorname{cem} 3 w x y^{2} \operatorname{sign}(E 4+x)-\operatorname{cem} 4 w x y^{2} \operatorname{sign}(E 4+x) \\
& +\operatorname{cem} 4 w x y^{2} \operatorname{sign}(E 5+x)-\operatorname{cem} 5 w x y^{2} \operatorname{sign}(E 5+x)
\end{aligned}
$$

